\title{
Evaluation of Metallo- $\beta$-Lactamase-Production and Carriage of bla-vim Genes in Pseudomonas aeruginosa Isolated from Burn Wound Infections in Isfahan
}

\author{
Mahmood Saffari, ${ }^{1}$ Farzaneh Firoozeh,,${ }^{2,}$ Mohammad Pourbabaee, ${ }^{2}$ and Mohammad Zibaei ${ }^{3}$ \\ ${ }^{1}$ Trauma Research Center, Kashan University of Medical Sciences, Kashan, IR Iran \\ ${ }^{2}$ Department of Microbiology and Immunology, School of Medicine, Kashan University of Medical Sciences, Kashan, IR Iran \\ ${ }^{3}$ Department of Parasitology and Mycology, School of Medicine, Alborz University of Medical Sciences, Karaj, IR Iran \\ "Corresponding author: Farzaneh Firoozeh, Department of Microbiology and Immunology, P. O. Box: 87159-88141, Kashan, IR Iran. Tel/Fax: +98-3155540021, E-mail: \\ ffiroozeh@ut.ac.ir
}

Received 2015 November 03; Revised 2015 December 25; Accepted 2016 January 01.

\begin{abstract}
Background: Metallo- $\beta$-lactamase-production among Gram-negative bacteria, including Pseudomonas aeruginosa, has become a challenge for treatment of infections due to these resistant bacteria.

Objectives: The aim of the current study was to evaluate the metallo- $\beta$-lactamase-production and carriage of bla-viM genes among carbapenem-resistant $P$. aeruginosa isolated from burn wound infections.

Patients and Methods: A cross-sectional study was conducted from September 2014 to July 2015. One hundred and fifty P. aeruginosa isolates were recovered from 600 patients with burn wound infections treated at Imam-Musa-Kazem Hospital in Isfahan city, Iran. Carbapenem-resistant $P$. aeruginosa isolates were screened by disk diffusion using CLSI guidelines. Metallo- $\beta$-lactamase-producing $P$. aeruginosa isolates were identified using an imipenem-EDTA double disk synergy test (EDTA-IMP DDST). For detection of MBL genes including bla-viM-1

Results: Among the 150 P. aeruginosa isolates, 144 (96\%) were resistant to imipenem by the disk diffusion method, all of which were identified as metallo- $\beta$-lactamaseproducing P. aeruginosa isolates by EDTA-IMP DDST. Twenty-seven (18\%) and 8 (5.5\%) MBL-producing P. aeruginosa isolates harbored bla-viM-1 and bla-viM-2 genes, respectively. Conclusions: Our findings showed a high occurrence of metallo- $\beta$-lactamase production among $P$. aeruginosa isolates in burn patient infections in our region. Also, there are $P$. aeruginosa isolates carrying the $b_{l} a_{-\mathrm{VIM}_{-1}}$ and $b l a_{-\mathrm{VIM}-2}$ genes in Isfahan province.
\end{abstract}

Keywords: Burn Patients, Metallo- $\beta$-Lactamase, bla $_{\mathrm{VIM}-1}$, bla $_{\mathrm{VIM}-1}$, Pseudomonas aeruginosa

\section{Background}

Burn wounds are major public health problems all over the world. Infection is one of the most complicated issues in burn patients, because the skin, a barrier against microbes, has been destroyed and the immunity agents cannot reach the sites of infection. There is a correlation between the severity of infection and the extent of the burn (1). It has been estimated that about $75 \%$ of burn-related mortality involves infections $(2,3)$. The rate of nosocomial infections among patients hospitalized in burn units is high, and P. aeruginosa has been documented as the most prevalent isolated bacteria in these patients $(4,5)$. Pseudomonas aeruginosa is characterized by an innate resistance to multiple antimicrobial agent as well as by acquired multidrug resistance ability. Due to multi-drug resistant $P$. aeruginosa isolates, treating these patients' burn wound infections is challenging (6). Carbapenems are a group of $\beta$-lactams that show resistance to hydrolysis by extended-spectrum $\beta$-lactamases (ESBLs), so are used as the drug of choice for treatment of infections caused by ESBL-positive Gram negative bacteria (7). Broad spectrum activity as well as these abilities have led to increased used of carbapenems, especially imipenem, for treatment of nosocomial infections (8). Unfortunately, the emergence of metallo- $\beta$-lactamase (MBL), the enzyme that inactivates carbapenems, has prevented the use of these antibiotics (9). In a study conducted at Ghotbeddin Burn hospital in Shiraz, 55 (20.4\%) MBL-producing P. aeruginosa were identified among $270 P$. aeruginosa isolated patients hospitalized in the burn unit (1). MBLs are categorized as Ambler class B (10). These $\beta$-lactamase groups of enzymes have resistance to all $\beta$-lactamase inhibitors, and due to the presence of zinc ion in their active sites, are inactivated by chelating agents such as ethylene diamine tetra acetic acid (EDTA) (9). Metallo- $\beta$-lactamase-production is encoded by either chromosomal genes or genes are inserted in mobile genetic elements, which especially facilitate the spread of these genes among Gram negative bacteria (9). Recently, several new MBL codes such as VIM have been documented in $P$. aeruginosa isolates (7). The three main clusters of VIM MBL have been reported, including VIM-1, VIM-2, and VIM-7 (7), and the VIM type metallo- $\beta$-lactamase genes are shown to encode by inserted cassettes in the mobile integrons (7, 11). The rate of the infections caused by $P$. aeruginosa in the burn unit of Imam-Musa-Kazem hospital in Isfahan was 
high; therefore rapid identification of MBL-producing $P$. aeruginosa strains is important.

\section{Objectives}

In the present study we determined the metallo- $\beta$ lactamase-production and carriage of bla $_{\text {-VIM }}$ genes among carbapenem-resistant $P$. aeruginosa isolates in burn patients in Isfahan Province.

\section{Patients and Methods}

\subsection{Subjects}

This cross-sectional study was conducted on 600 patients, who were hospitalized in the burn unit of Imam-Musa-Kazem university Hospital in Isfahan between September 2014 and July 2015.

\subsection{Bacterial strains}

For sampling, wound swabs were obtained from the burn patients and $P$. aeruginosa was identified by standard microbiological methods including Gram stains, culture on mediums such as blood agar, MacConkey agar, triple sugar iron agar (TSI), cetrimide agar (Merck, UK), oxidase (Sigma, USA), catalase, oxidative/fermentative (OF), indole, methyl red (MR), Voges-Proskauer (VP) tests (Merck, UK), and growth at $42^{\circ} \mathrm{C}$.

\subsection{Screening of Carbapenem-Resistant and MBL Producing Pseudomonas aeruginosa Isolates}

The disk diffusion method was used according to the clinical and laboratory standards institute (CLSI) guidelines for detection of carbapenem-resistant $P$. aeruginosa isolates, and the imipenem $(10 \mu \mathrm{g})$ and meropenem $(10 \mu \mathrm{g})$ disks were purchased from Mast, UK Company (12). A P. aeruginosa standard strain (ATCC 27853) was used for quality control in susceptibility testing. Phenotypic screening for MBL producers $P$. aeruginosa isolates was performed using an EDTA double disk synergy test (EDTA-IMP DDST) in which imipenem resistant isolates selected by the disk diffusion method were examined. Briefly, a colony of each $P$. aeruginosa strain was suspended in Mueller-Hinton (MH) broth to $106 \mathrm{CFU} / \mathrm{mL}$. after being spread on an $\mathrm{MH}$ agar plate with a cotton swab. Two commercial disks containing $10 \mu \mathrm{g}$ imipenem with $(10 \mu \mathrm{g} / 750 \mu \mathrm{g})$ and without EDTA were placed on the center of the plate at a distance of $25 \mathrm{~mm}$ from each other, and were incubated at $37^{\circ} \mathrm{C}$ overnight. After overnight incubation, the presence of $\mathrm{a} \geq 7 \mathrm{~mm}$ synergistic inhibition zone around the IMP-EDTA disk in comparison to the IMP disk was interpreted as positive (13).

\subsection{Polymerase Chain Reaction Detection of Genes for MBLs}

DNA from each $P$. aeruginosa isolate was extracted by the boiling method. Templates of the DNA were stored at $-20^{\circ} \mathrm{C}$ until PCR amplification was performed. MBLproducing $P$. aeruginosa strains which were identified by a DDST confirmatory test were amplified by the PCR method, using bla -VIM-1 $_{\text {and }}$ bla-VIM-2 specific primers (Table 1) for amplification of $261 \mathrm{bp}$ and $801 \mathrm{bp}$ fragments, respectively (14).

Reactions were carried out using a thermo cycler (Eppendorf master cycler $^{\circledR}, \mathrm{MA}$ ) in the PCR conditions previously described (14). For confirmation, some PCR products were sent elsewhere for sequencing (Bioneer, South Korea). The nucleotide sequences were analyzed by the program Chromas Pro version1.7.5 Technelysium (www.technelysium.com.au), and were aligned with GenBank to check the identity of each sequence against those deposited in GenBank.

\subsection{Ethical Considerations}

Each patient's demographic information was obtained by a survey questionnaire, and a written informed consent was received from all patients or their parents. The study design, including its ethical aspects, was reviewed and approved by the ethics committee of Kashan University of Medical Sciences (No. 4380).

\subsection{Statistical Analysis}

The statistical analysis of data was conducted using SPSS software version 16 (SPSS, Inc.). Differences by the $\chi^{2}$ test were considered statistically significant if the $P$ value was less than $0.05(\mathrm{P}<0.05)$.

\section{Results}

Of 600 burn patients, who had been hospitalized in the burn unit of Imam-Musa-Kazem university hospital in Isfahan, a total of $150 P$. aeruginosa isolates were recovered. The patients with isolates included 69 (46.0\%) females and 81 (54.0\%) males, and their ages ranged between 1 and 72 years old (Table 2 ). Of the $150 P$. aeruginosa isolates identified by standard biochemical tests, 144 (96.0\%) and 144 (96.0\%) showed resistance to imipenem and meropenem, respectively, by the disk diffusion method. An EDTA-IMP DDST phenotypic confirmatory test revealed that $100 \%$ of the imipenem resistant $P$. aeruginosa isolates were metallo$\beta$-lactamase producing (Figure 1).

The finding of the PCR assays showed that of 144 MBLproducing $P$. aeruginosa isolates, 27 (18\%) and 8 (5.5\%) were positive for the bla-viM-1 and bla-vim-2 genes, respectively (Figure 2). The nucleotide sequences of the PCR products of the bla-viM-1 and bla-viM-2 genes were identical to those deposited in GenBank. 
Table 1. Primers Used for Polymerase Chain Reaction and Sequencing

\begin{tabular}{ccc}
\hline Primer & Target Gene & Sequence $\left(\mathbf{5}^{\prime} \rightarrow \mathbf{3}\right)$ \\
\hline VIM-1 F & $b l a_{\mathrm{VIM}-1}$ & AGT GGT GAG TAT CCG ACA G \\
VIM-1 R & $b l a_{\mathrm{VIM}-1}$ & ATG AAA GTG CGT GGA GAC \\
VIM-2 F & $b l a_{\mathrm{VIM}-2}$ & ATG TTC AAA CTT TTG AGT AAG \\
VIM-2 R & $b l a_{\mathrm{VIM}-2}$ & CTA CTC AAC GAC TGA GCG \\
\hline
\end{tabular}

Table 2. Association Between Burn Patients' Demographic Characteristics and bla $a_{\mathrm{VIM}}$ Genes Carriage Among Pseudomonas aeroginosa Isolates

\begin{tabular}{|c|c|c|c|c|c|c|c|}
\hline \multirow[t]{2}{*}{ Factor } & \multicolumn{2}{|c|}{$b^{\prime l a} a_{\mathrm{VIM}-1}$} & \multirow[t]{2}{*}{ PValue } & \multicolumn{2}{|c|}{$b^{b l a} a_{\mathrm{VIM}-2}$} & \multirow[t]{2}{*}{ PValue } & \multirow[t]{2}{*}{ Total, No. (\%) } \\
\hline & Positive, No. (\%) & Negative, No. (\%) & & Positive, No. (\%) & Negative, No. (\%) & & \\
\hline \multicolumn{8}{|l|}{ Sex } \\
\hline Male & $20(13.3)$ & $61(40.7)$ & 0.374 & $4(2.7)$ & $77(51.3)$ & 0.449 & $81(54.0)$ \\
\hline Female & $7(4.7)$ & $62(41.3)$ & & $4(2.7)$ & $65(43.3)$ & & $69(46.0)$ \\
\hline Less than 30 & $5(3.3)$ & $25(16.7)$ & 0.812 & $1(0.7)$ & $29(19.3)$ & 1 & $30(20.0)$ \\
\hline $30-49$ & $21(14.0)$ & $90(60.0)$ & & $3(2.0)$ & $108(72.0)$ & & $111(74.0)$ \\
\hline More than 50 & $1(0.7)$ & $8(5.3)$ & & $4(2.7)$ & $5(3.3)$ & & $9(6.0)$ \\
\hline \multicolumn{8}{|l|}{ Burn level (38.85 \pm 7.58$)$} \\
\hline Equal and less than 35 & $7(4.7)$ & $24(16.0)$ & 0.041 & $3(2.0)$ & $28(18.7)$ & 0.032 & $31(20.7)$ \\
\hline \multicolumn{8}{|l|}{ Burn cause } \\
\hline Fire and flame & $13(8.7)$ & $49(32.6)$ & 0.161 & $4(2.7)$ & $58(38.6)$ & 0.212 & $62(41.3)$ \\
\hline Chemical & $5(3.3)$ & $14(9.4)$ & & 0 & $19(12.7)$ & & $19(12.7)$ \\
\hline Boiled water and hot liquids & $9(6.0)$ & $60(40.0)$ & & $4(2.7)$ & $65(43.3)$ & & $69(46.0)$ \\
\hline
\end{tabular}

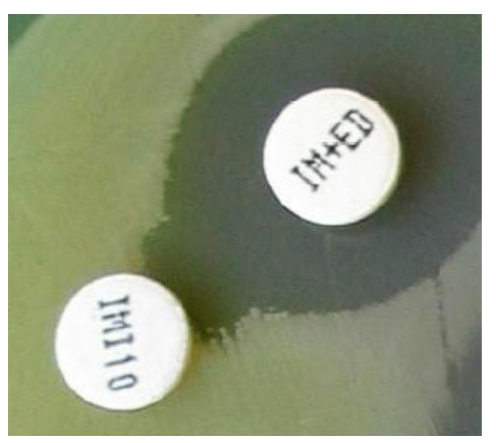

Figure 1. Metallo- $\beta$-Lactamase Producing Pseudomonas aeruginosa Isolates Identified By a Imipenem-EDTA Double Disk Synergy Test(EDTA-IMP DDST) Phenotypic Confirmatory Test

\section{Discussion}

Pseudomonas aeruginosa is documented as one of the most frequently isolated bacteria in nosocomial infec- tions, especially in burn units (3). Severe antimicrobial resistance in this bacterium has complicated the treatment of infections caused by drug-resistant $P$. aeruginosa strains among burn patients (15). Recently, the increase of carbapenem resistance in $P$. aeruginosa has been documented all over the world due to metallo- $\beta$-lactamase production, including the VIM type (16). This study revealed that the resistance rates to imipenem and meropenem were very high (96\%). Farajzadeh Sheikh et al. (15) reported an imipenem resistance rate of $58.7 \%$ among $P$. aeruginosa isolates in Ahwaz, which was lower than our resistance rate. However, Anvarinejad and colleagues' (1) study, also conducted in Ahwaz, was in agreement with our study: 98.1\% of MBL-producing P. aeruginosa strains, which were isolated from hospitalized patients in a burn unit showed resistance to imipenem. Another report, in contrast with our study, found a resistance rate of $21 \%$ to imipenem among $P$. aeruginosa samples from burn patients in Kurdistan (3). As carbapenems, including imipenem, are the 
Figure 2. Amplification of bla $_{\mathrm{VIM}-1}$ and $b l a_{\mathrm{VIM}-2}$ Genes in Imipenem Resistant MBL Positive Pseudomonas aeruginosa Isolates
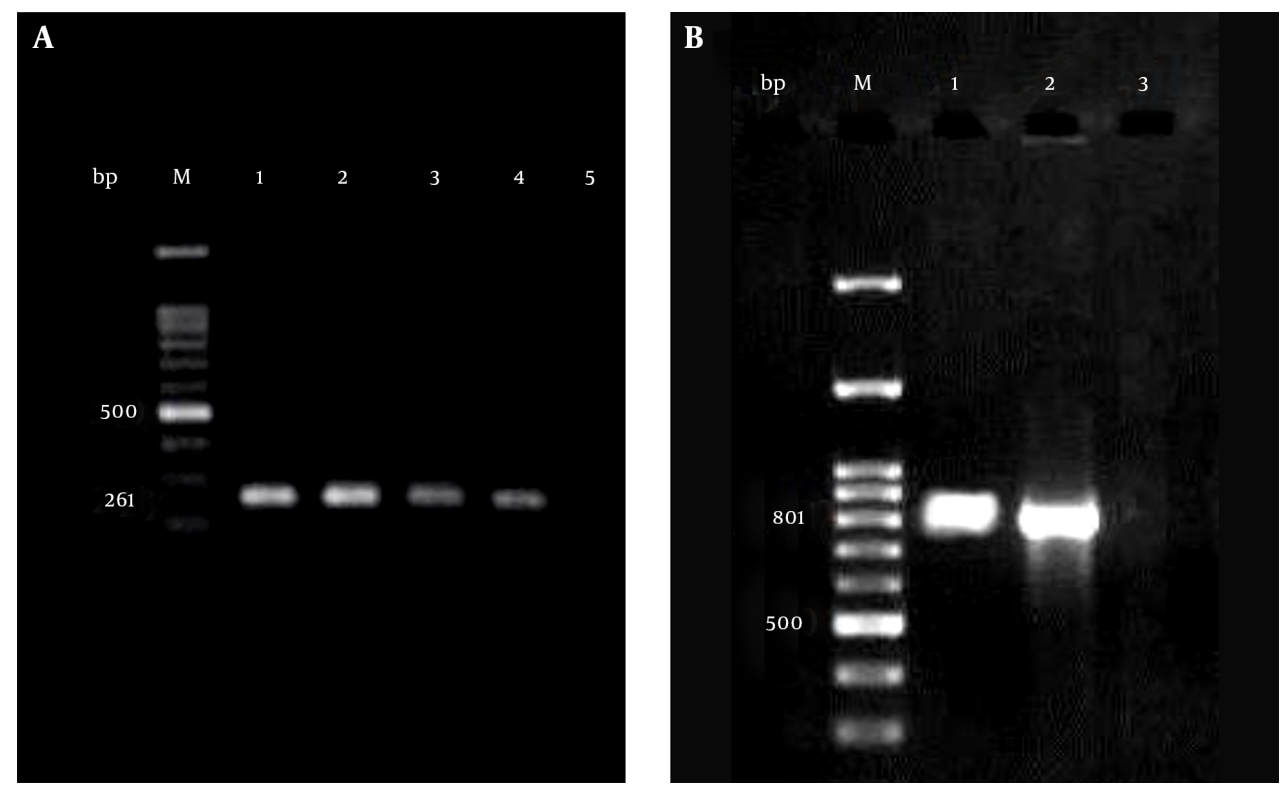

M lanes, 100-bp DNA ladder as the molecular size marker; A, lane 1, positive control; lanes 2 - 4, bla $a_{\mathrm{VIM}-1}$ positive Pseudomonas aeruginosa isolates; lane 5, negative control; B, lane 1, positive control; lane 2, bla $a_{\mathrm{VIM}-2}$ positive Pseudomonas aeruginosa isolate; lane 3, negative control.

drugs of choice for treatment of infections caused by MDR$P$. aeruginosa strains, this result in particularly alarming for burn patients with immune-compromised defenses. The high resistance rates to carbapenems may be relatively imprecise because they were not confirmed by confirmatory tests such as an E-test. The difference in resistance rates to imipenem in P. aeruginosa isolates from burn patients in Ahwaz, Isfahan, and Kurdistan could be due to frequent use of this antibiotic and the occurrence of high selective pressure in Ahwaz and Isfahan, and fewer patients' access to antibiotics due to economic problems in Kurdistan.

MBL-producing $P$. aeruginos a have been isolated from hospitalized patients increasingly, and isolates that produce these families of enzymes have been documented as one cause of prolonged nosocomial infections (17). Here we showed that all imipenem-resistant $P$. aeruginosa isolates had the ability of MBL-production. In Kurdistan and Ahwaz 22\% and $19.5 \%$ of P. aeruginosa isolates among burn patients were identified as MBL-producing isolates (3). In a study conducted by Farajzadeh Sheikh et al. (15) in Ahwaz among P. aeruginosa isolates in burn and nonburn patients, all of the imipenem-resistant isolates were MBL E-test positive, which correlates with our results, although it would be better if the MBL-production among our imipenem resistant $P$. aeruginosa isolates were confirmed by an MBL E-test as well. The EDTA-IMP DDST test and sodium mercaptoacetic acid (SMA)-IMP DDST revealed that $98.1 \%$ of imipenem-resistant $P$. aeruginosa and $78.9 \%$ of $P$. aeruginosa isolates were positive for production of metallo- $\beta$-lactamase enzymes in Brazil and Japan, respectively $(8,14)$. This finding shows that the prevalence of carbapenemases among nosocomial isolates of $P$. aeruginosa is high, especially in burn units.

It has been estimated that more than $40 \%$ of imipenem resistance in P. aeruginosa (IRPA) is due to MBL production, although enzyme types vary in different regions (18, 19). In the present study, $18 \%$ and $5.5 \%$ of MBL positive isolates were VIM-1 and VIM-2 MBL types, respectively. Similar to our finding, 19.3\% of MBL-producing P. aeruginosa isolates in burn patients in Ahwaz were positive for bla.VIM genes (7). In a study in Brazil, 19\% of IRPA isolates which were MBL-positive were shown to carry $b a_{\text {-VIM-2 }}$, whereas $b l a_{\text {-VIM-1 }}$ was not detected among them (8). The fact that the $b_{l a} a_{\text {-IIM-2 }}$ gene was detected in $48.8 \%$ of IRPA clinical isolates in Romania (20) shows that MBL type enzymes can vary geographically. Another interesting finding was that the remaining 109 IRPA isolates, which were MBL positive by a phenotypic test, proved to be negative for bla $_{\text {-VIM-1 }}$ and

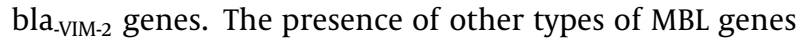
could be the reason.

Overall, the findings of the current study showed that the resistance rate to carbapenems, as the last-resort therapy in infections due to MDR P. aeruginosa strains among burn patients, is high. Therefore, in these patients a reg- 
ular surveillance program for controlling infection and in vitro testing of antibiotics before empiric treatment could be helpful to control MDR-Pseudomonas aeruginosa infections in the burn units. Also, a high occurrence of MBL production among imipenem-resistant $P$. aeruginosa isolates in burn patients was seen. Further studies using more accurate confirmatory tests in our region are recommended.

\section{Acknowledgments}

We are grateful to Mr. Radan for collecting the samples and helping with technical assistance.

\section{Footnote}

Authors' Contributions: Farzaneh Firoozeh contributed to the study design, study management, supervision, and prepared and wrote the manuscript. Mohammad Pourbabaee performed the sampling, processing, and conventional and molecular procedures. Mohammad Zibaei provided advice, and read and arranged the final manuscript. Mahmood Saffari provided advice.

\section{References}

1. Anvarinejad M, Japoni A, Rafaatpour N, Mardaneh J, Abbasi P, Amin Shahidi M, et al. Burn Patients Infected With Metallo-Beta-LactamaseProducing Pseudomonas aeruginosa: Multidrug-Resistant Strains. Arch Trauma Res. 2014;3(2):18182. doi: 10.5812/atr.18182. [PubMed 25147779].

2. Poole K. Multidrug efflux pumps and antimicrobial resistance in Pseudomonas aeruginosa and related organisms. $J$ Mol Microbiol Biotechnol. 2001;3(2):255-64. [PubMed: 11321581].

3. Kalantar E, Torabi V, Salimizand H, Soheili F, Ramezanzadeh R. Incidence and Susceptibility Pattern of Metallo-Beta-Lactamase Producers Among Pseudomonas aeruginosa Isolated From Burn Patients at Kurdistan Province. Jundishapur J Microbiol. 2012;5(3):507-10. doi: 10.5812/jjm.3664

4. Agnihotri N, Gupta V, Joshi RM. Aerobic bacterial isolates from burn wound infections and their antibiograms-a five-year study. Burns. 2004;30(3):241-3. doi:10.1016/j.burns.2003.11.010.[PubMed:15082351].

5. Weldhagen GF, Poirel L, Nordmann P. Ambler class A extendedspectrum beta-lactamases in Pseudomonas aeruginosa: novel developments and clinical impact. Antimicrob Agents Chemother. 2003;47(8):2385-92. [PubMed: 12878494].

6. Porras-Gomez M, Vega-Baudrit J, Nunez-Corrales S. Overview of Multidrug-Resistant Pseudomonas aeruginosa; and Novel Therapeutic Approaches. J Biomater Nanobiotechnol. 2012;03(04):519-27.
7. Khosravi AD, Mihani F. Detection of metallo-beta-lactamaseproducing Pseudomonas aeruginosa strains isolated from burn patients in Ahwaz, Iran. Diagn Microbiol Infect Dis. 2008;60(1):125-8. doi: 10.1016/j.diagmicrobio.2007.08.003. [PubMed: 17900848].

8. Franco MR, Caiaffa-Filho HH, Burattini MN, Rossi F. Metallo-betalactamases among imipenem-resistant Pseudomonas aeruginosa in a Brazilian university hospital. Clinics (Sao Paulo). 2010;65(9):825-9. [PubMed: 21049207].

9. Wirth FW, Picoli SU, Cantarelli VV, Goncalves AL, Brust FR, Santos LM, et al. Metallo-beta-lactamase-producing Pseudomonas aeruginosa in two hospitals from southern Brazil. Braz J Infect Dis. 2009;13(3):170-2. [PubMed: 20191191].

10. Doosti M, Ramazani A, Garshasbi M. Identification and characterization of metallo-beta-lactamases producing Pseudomonas aeruginosa clinical isolates in University Hospital from Zanjan Province, Iran. Iran Biomed J. 2013;17(3):129-33. [PubMed: 23748890].

11. Yatsuyanagi J, Saito S, Harata S, Suzuki N, Ito Y, Amano K, et al. Class 1 integron containing metallo-beta-lactamase gene blaVIM-2 in Pseudomonas aeruginosa clinical strains isolated in Japan. Antimicrob Agents Chemother. 2004;48(2):626-8. [PubMed: 14742222].

12. Clinical and Laboratory Standards Institute . Performance Standards for antimicrobial susceptibility testing 21th information supplement. USA: CLSI; 2012.

13. Lee K, Lim YS, Yong D, Yum JH, Chong Y. Evaluation of the Hodge test and the imipenem-EDTA double-disk synergy test for differentiating metallo-beta-lactamase-producing isolates of Pseudomonas spp. and Acinetobacter spp. J Clin Microbiol. 2003;41(10):4623-9. [PubMed: 14532193].

14. Shibata N, Doi Y, Yamane K, Yagi T, Kurokawa H, Shibayama K, et al. PCR typing of genetic determinants for metallo-beta-lactamases and integrases carried by gram-negative bacteria isolated in Japan, with focus on the class 3 integron. J Clin Microbiol. 2003;41(12):5407-13. [PubMed: 14662918].

15. Farajzadeh Sheikh A, Rostami S, Jolodar A, Tabatabaiefar MA, Khorvash F, Saki A, et al. Detection of Metallo-Beta Lactamases Among Carbapenem-Resistant Pseudomonas aeruginosa.Jundishapur JMicrobiol. 2014;7(11):12289. doi: 10.5812/jjm.12289. [PubMed: 25774271].

16. Touati M, Diene SM, Dekhil M, Djahoudi A, Racherache A, Rolain JM. Dissemination of a class I integron carrying VIM-2 carbapenemase in Pseudomonas aeruginosa clinical isolates from a hospital intensive care unit in Annaba, Algeria. Antimicrob Agents Chemother. 2013;57(5):2426-7. doi: 10.1128/AAC.00032-13. [PubMed: 23459493].

17. Alaghehbandan R, MacKay Rossignol A, Rastegar Lari A. Pediatric burn injuries in Tehran, Iran. Burns. 2001;27(2):115-8. [PubMed: 11226645].

18. Livermore DM. Of Pseudomonas, porins, pumps and carbapenems. $J$ Antimicrob Chemother. 2001;47(3):247-50. [PubMed:11222556].

19. Livermore DM. Interplay of impermeability and chromosomal betalactamase activity in imipenem-resistant Pseudomonas aeruginosa. Antimicrob Agents Chemother. 1992;36(9):2046-8. [PubMed: 1329641].

20. Mereuta AI, Badescu AC, Dorneanu OS, Iancu LS, Tuchilus CG. Spread of VIM-2 metallo-beta-lactamase in Pseudomonas aeruginosa and Acinetobacter baumannii clinical isolates from Iași, Romania. RRLM. 2013;21(4):423-30. 